\title{
Evaluation of diagnostic value of conventional and color Doppler ultrasound with elastography strain ratios in differentiation between benign and malignant lymph nodes
}

\author{
Deniz Özel $\left.\right|^{1 A, B, C, D, E, F,}$, Betül Duran Özel|2E,F \\ ${ }^{1}$ Clinic of Radiology, Okmeydani Education and Research Hospital, İstanbul, Turkey \\ ${ }^{2}$ Clinic of Radiology, Şişli Hamidiye Etfal Education and Research Hospital, İstanbul, Turkey
}

\section{Abstract}

Purpose: The aim of this study was to evaluate the diagnostic value of conventional ultrasonography (USG) and color Doppler ultrasonography (CDU) with elastography strain ratios (ESR) in discrimination between benign and malignant lymphatic nodes.

Material and methods: Two hundred and forty-seven patients (252 lymph nodes) were included in this study. USG and CDU with ESR were performed. Materials were obtained by using fine-needle or vacuum-assisted aspiration methods depending on the site and condition of lymph nodes. The $\chi^{2}$ test and Student's $t$-test were used for comparisons.

Results: Ninety-two of 252 (36.5\%) lymph nodes were malignant, and 160 (63.5\%) were benign. Short axis, S/L ratio, presence or absence of the fatty hilum and extra-hilar vascularity were valuable, statistically significant indicators of malignancy. In contrast, long axis and ESR were not significant indicators of malignancy. The short axis cut-off value was calculated to be $12 \mathrm{~mm}$ and the S/L ratio cut-off value was calculated to be 1 .

Conclusions: Long axis of lymph nodes and strain ratios cannot be used as malignancy criteria, and their use is controversial despite the fact that some studies have found them valuable. On the other hand, our results support common knowledge that the short axis, S/L ratio, absence of the fatty hilum, and extrahilar vascularity are significant indicators. Key words: elasticity imaging techniques, lymph nodes, ultrasonography.

\section{Introduction}

During follow-up or even routine examinations, gray-scale ultrasonography (USG) combined with color Doppler ultrasonography (CDU) can often detect lymph nodes that appear pathological due to an unknown cause. Atypical lymph nodes can be detected in any area, including healthy individuals, in acute or chronic infections, and metabolic and rheumatoid diseases. However, when the radiologist defines lymph nodes as "atypical", clinicians may be prone to perform additional and sometimes unnecessary invasive procedures such as needle or excisional biopsy.

According to the literature, abnormal lymph nodes are those spherical in shape, with a short-to-long-axis ratio $(\mathrm{S} / \mathrm{L}$ ratio or roundness index $)>0.5$, with absent central echoic hilum, an irregular cortex, ill-defined borders, or presence of vascular signals outside the hilum [1].

Elastography, a recently developed diagnostic method, can contribute additional information to conventional USG findings. Two kinds of elasticity assessments can

\footnotetext{
Correspondence address:

Deniz Özel, Okmeydani Education and Research Hospital, Kaptanpasa Mah. Darülaceze Cad. No: 2734384 Okmeydanı - Şişli, İstanbul, Turkey,

e-mail: denizozel34@hotmail.com
}

Authors' contribution:

A Study design - B Data collection · C Statistical analysis · D Data interpretation - E Manuscript preparation · $F$ Literature search · G Funds collection 
be performed other than the shear-wave approach. First, visual scoring of colors within and around the lesions using a 4 - or 5-point elastographic scale, measuring the relative proportion of the intra-lesion low strain areas, can be used. In such assessments, the stiffer the tissue, the higher is the score. Second, two regions of interest (ROIs) can be placed over the target region and adjacent reference tissue consisting of normal muscle or subcutaneous fat. The strain ratio is then automatically computed by the USG device. According to some studies, strain ratio values above a certain cut-off value indicate an increased likelihood of malignancy [2-6].

In daily practice, interventional radiologists often see many pathological or suspicious lymph nodes in a single examination, and they may need to choose the most appropriate candidates for further workup.

The aim of this study was to evaluate conventional USG and CDU findings with elastography strain ratios (ESR) that could predict histological features, benign or malignant, of lymph nodes.

\section{Material and methods}

We prospectively included 247 patients of our institute and 252 lymph nodes after performing USG-guided biopsies between September 2014 and December 2016.

Twenty-seven lymph nodes were excluded due to inadequate material and unreliable results.

Before the procedure, all patients were questioned for contraindications such as anticoagulant treatment or anxiety that could interfere with biopsy.

After sterilization, topical lidocaine solution (Vemcaine Pump Spray 10\%) and 5-10 ml of bupivacaine solution (Bustesin solution 0.5\%) were used as anesthetics to reduce the pain at the procedure site.

\section{Imaging procedure}

USG was performed with the Aplio 500 device (Toshiba Medical Systems Europe), with a 7.2-14 MHz linear probe. The probe was covered with a sterile instrument. Samples were obtained by using fine-needle or vacuum-assisted aspiration depending on the site and condition of lymph nodes. Fine-needle biopsies were performed with 22-G needles, and vacuum-assisted biopsies were performed with $18-\mathrm{G}$ needles.

The vacuum-assisted method was preferred if nodes contained pure solid material and were placed far away from neurovascular structures.

All procedures were performed by the same interventional radiologist with 4 years of experience.

After hemostasis was confirmed, sings of bleeding under the skin were controlled. All patients were observed for a short period of time.

After the histopathological results were obtained, conventional ultrasound findings were evaluated for compar- ison. They included long axis, short axis, short axis/long axis ratio $(\mathrm{S} / \mathrm{L})$, presence or absence of the fatty hilum, extrahilar vascularity on CDU imaging, and ESR.

\section{Statistical analysis}

IBM SPSS Statistic version 22 was used. The $\chi^{2}$ test and the Student's $t$-test were used for comparisons.

ROC analysis was used for calculating cut-off values.

\section{Ethical statement}

This study was approved by our Institute's Research Ethics Committee (15.12.2015 no: 275), and informed consents were obtained from all patients prior to enrollment.

All procedures performed in studies involving human participants were in accordance with the ethical standards of the institutional and national research committee and with the 1964 Helsinki declaration and its later amendments or comparable ethical standards.

\section{Informed consent}

Informed consent was obtained from all individual participants included in the study.

\section{Results}

Fifty-three of 247 (21.5\%) patients were male and 194 (79.5\%) were female.

The patients were aged between $10-86$ years (mean: 50.15 years).

Ninety-two of 252 (36.5\%) lymph nodes were malignant, and 160 (63.5\%) were benign.

Histopathological results are shown in Table 1.

Table 1. Distribution of benign and malignant histopathological results

\begin{tabular}{|l|l|c|c|}
\hline Benign (160) & Non neoplastic lymphoid & 104 & 65.0 \\
& $\begin{array}{l}\text { tissue } \\
\text { Reactive hyperplasia }\end{array}$ & 38 & 23.8 \\
& Granulomatous & 13 & 8.1 \\
& lymphadenitis & & \\
& Necrotic lymphoid tissue & 5 & 3.1 \\
\hline Malignant (92) & Carcinoma from unknown & 34 & 37.0 \\
& origin & & \\
& Breast ca metastasis & 29 & 31.5 \\
& Lymphoma & 12 & 13.0 \\
& Thyroid ca & 9 & 10.0 \\
& Nasopharynx ca & 4 & 4.3 \\
& Sarcoma & 2 & 2.2 \\
& Lung ca & 1 & 1.1 \\
& Anaplastic ca & 1 & 1.1 \\
\hline & Total & 252 & \\
\hline
\end{tabular}


Table 2. Malignancy ratio of the lymph nodules according to their regions

\begin{tabular}{|l|c|c|c|}
\hline Region & No of nodules & $\begin{array}{c}\text { Malignant } \\
\text { nodules }\end{array}$ & $\begin{array}{c}\text { Malignancy } \\
\text { ratio }\end{array}$ \\
\hline Neck all levels & 126 & 34 & 27.0 \\
\hline Axillary & 116 & 55 & 47.4 \\
\hline Inguinal & 10 & 3 & 30.0 \\
\hline Total & 252 & 92 & 36.5 \\
\hline
\end{tabular}

Distribution of malignancy ratios according to the regions are shown in Table 2.

Short axis, S/L ratio, absence of echogenic hilum, and extra-hilum vascularity on power Doppler imaging were valuable, statistically significant features of malignant changes $(p<0.01)$.

The short axis cut-off value was calculated to be $12 \mathrm{~mm}$, with sensitivity of $65 \%$ and specificity of $77 \%$.

The S/L ratio cut-off value was calculated to be 1 , with sensitivity of $100 \%$ and specificity of $97.5 \%$.

In contrast, long axis and ESR were not significant features of malignancy.

Table 3 shows imaging features of benign and malignant lymph nodes.

\section{Discussion}

Many studies have evaluated sonographic features of lymph nodes, and the majority of them prefer evaluations based on lymph nodes placement site. The main advantage of this method is that lymph nodes may show regional characteristics typical for tumors that infiltrate them. Metastatic nodes tend to be hypoechoic compared with adjacent muscles $[7,8]$. However, metastatic nodes from the papillary carcinoma of the thyroid gland are usually hyperechoic. This hyperechogenicity of lymph nodes is believed to result from intranodal deposition of thyroglobulin, which originates from the thyroid primary tumor [9]. In our study, we tried to define the features of malignant lymph nodes.

The overall malignancy ratio was $36.5 \%$, and it was higher than the ratio reported in similar articles (20-25\%). This study was based on data from oncological inpatients, and the majority of lymph nodes were studied in patients who remained under long-term oncological follow-up. This can account for the high malignancy ratio in our study. Our patients were predominantly female (79.5\%). In our institute, there are many patients with thyroid and breast diseases that are diagnosed more commonly in females.

At first glance, common cancers like breast and thyroid cancers were frequent in our study. Biopsies were performed in the neck, axillary, and inguinal regions, and those cancers tend to spread within these regions as well.

The majority of benign lesions were diagnosed as non-neoplastic lymphatic tissue. This is the name given by our pathologist to benign lymph nodes. There were also other findings, such as reactive hyperplasia, lymphadenitis, and necrotic lymph tissue, but they had a lower prevalence.

First, dimensions of the nodes were evaluated. The difference between the long axis of benign and malignant lymph nodes was not statistically significant. Regardless of the etiology, infiltrated lymph nodes were enlarged. But for the differentiation between benign and malignant lymph nodes, the longest dimension of the nodule was not a valuable indicator because benign causes may also lead to enlargement (e.g. inflammation). Additionally, lymph nodes with micrometastases may be small.

Malignant lesions diagnosed in the axillary region were mostly breast carcinoma. In the neck, there were various malignant lesions on histopathology. The majority of them were thyroid carcinoma metastases and lymphomas, probably because of proximity. Finally, in the inguinal region, the majority of the malignant lesions were lymphomas.

The short axis and S/L ratio were statistically significant indicators of malignancy. In our experience, one must be aware of malignancy if node enlargement is seen in the short axis with a high $\mathrm{S} / \mathrm{L}$ ratio. Malignant nodes tend to be spherical with $\mathrm{S} / \mathrm{L}$ ratio higher than 0.5 . The cut-off value was calculated to be 1 , with sensitivity of $100 \%$ and specificity of $97.5 \%$. This result indicates that the closer a lymph node is to be spherical, the closer it is to be malignant.

In our study, the mean short axis diameter was higher in malignant lymph nodes than in benign nodes, and the difference was statistically significant. This is in line with previous studies $[9,10]$. Metastatic lymph nodes are very cellular because of tumor cell infiltration. Tumor cells de-

Table 3. Imaging features of benign and malignant lymph nodes

\begin{tabular}{|c|c|c|c|}
\hline Imaging features & Benign lymph nodes $(n=160)$ & Malignant lymph nodes $(n=92)$ & $p$ \\
\hline \multicolumn{3}{|l|}{ Conventional ultrasound features } & 0.09 \\
\hline Long axis diameter (mm), mean (SD) & $22(6.75)$ & $22.37(11.35)$ & $<0.01$ \\
\hline Short axis diameter (mm), mean (SD) & $9.17(3.45)$ & $14.1(6.63)$ & $<0.01$ \\
\hline Ratio of short axis to long axis, mean (SD) & $0.48(0.17)$ & $0.67(0.18)$ & $<0.01$ \\
\hline Hilar loss, no (\%) of nodules & $31(17.8)$ & $64(68.3)$ & $<0.01$ \\
\hline Extrahilar vascularity at PD, no (\%) of nodules & $13(8.2)$ & $33(37.8)$ & 0.11 \\
\hline Strain ratio, mean (SD) & $2.94(3.61)$ & $3.93(3.71)$ & \\
\hline
\end{tabular}


stroy normal architecture of lymph nodes, thereby leading to changes in shape. Under normal conditions, most lymph nodes have an oval shape. After infiltration, tumor cells disorderly invade all parts of the node. With increasing cellularity, the node becomes spherical. Ying et al. [8] serially monitored the size of lymph nodes in patients with a known malignancy. An increase in nodal size on serial examinations is highly suspicious of metastases. In addition, a serial change in the size of metastatic nodes is useful for monitoring response to treatment. Based on ROC analysis, the cut-off value was calculated to be $12 \mathrm{~mm}$. Sensitivity and specificity values were $65 \%$ and $77 \%$, respectively.

Extrahilar vascularity was also evaluated. Malignant lymph nodes more often than benign nodes had extrahilar vascularity, and this difference was statistically significant. There are many articles that support this finding. In the neck, about $90 \%$ of lymph nodes with a maximum transverse diameter greater than $5 \mathrm{~mm}$ show a hilar vascularity.

Normal and reactive lymph nodes tend to demonstrate hilar vascularity or appear as avascular. However, metastatic lymph nodes usually have a peripheral or mixed (hilar and peripheral) vascularity $[11,12]$. Gupta et al. [9] found that $93.5 \%$ of reactive lymph nodes had a hilar vascularity, and $91.7 \%$ of metastatic lymph nodes had a peripheral vascularity $(n=64)$.

Subsequently, obliteration of the fatty hilum of lymph nodes was evaluated. The presence of an intranodal echogenic hilum was considered as a sign of benignity. In our study, the difference between the ratios of absence of the fatty hilum in benign and malignant nodes was statistically significant. Gronkiewicz et al. [13] found this feature as the only significant indicator in their study with 24 patients. On the other hand, Yeon Hwa Yoo et al. [14] did not find a statistically significant difference between malignant and benign nodes with respect to this feature in their study with 124 patients. Obliteration of the fatty hilum can be seen as the final step of cortical thickening. The fatty hilum can be infiltrated by inflammatory or tumor cells, and it is impossible to distinguish the hilum and the cortex of the node. Additionally, it is challenging to measure the real cortical thickness of the node. Therefore, we decided to evaluate the absence of the fatty hilum without cortical thickening to obtain clear results.

Finally, ESR ratios were compared. The mean strain ratio of malignant nodes was higher than the mean strain ratio of benign nodes. However, the difference was not statistically significant. It can be clearly seen that standard deviations were high when comparing strain ratios. Park et al. [15] used strain-wave elastography and did not find any contribution of elastography in comparison to conventional ultrasonography in a study including 104 nodes. That study evaluated axillary lymph nodes in terms of percentages of hard areas.

Strain ratio values are affected by the adjacent tissues and are highly user-dependent, which lowers the reliability of this method. To solve this problem, muscles were chosen as the reference, because muscles can be found easily at the procedure site that we focused on. On the other hand, there is a potential problem regarding muscle contractions, which can affect the stiffness. Therefore, one limitation of this study is that due to lack of appropriate equipment we were not able to use shear-wave elastography, which is newer and potentially more objective. We did not evaluate calcifications, central necrosis (cystic area), and CDU measurements of lymph flow. In our experience, central, cystic areas and calcifications are rarely seen in lymph nodes, and to see statistically significant effects, a larger group of patients is needed. This is another limitation of our study. Additionally, there are many color Doppler features that can be evaluated in separate studies.

\section{Conclusions}

In conclusion, we postulate that the long axis of the lymph node and strain ratios cannot be used as criteria of malignancy and their use is controversial despite the fact that

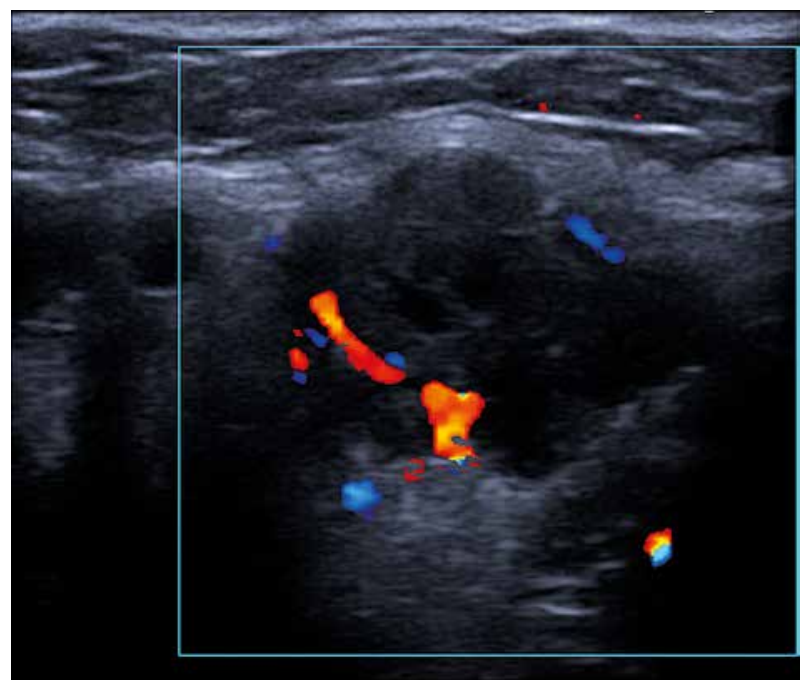

Figure 1. Malignant lymph node. A 58-year-old female. Submandibular region. Long axis: $20 \mathrm{~mm}$. Short axis: $16 \mathrm{~mm}$. S/L ratio: 0.8. Extrahilar vascularity. Pathology: Metastasis of nasopharyngeal cancer

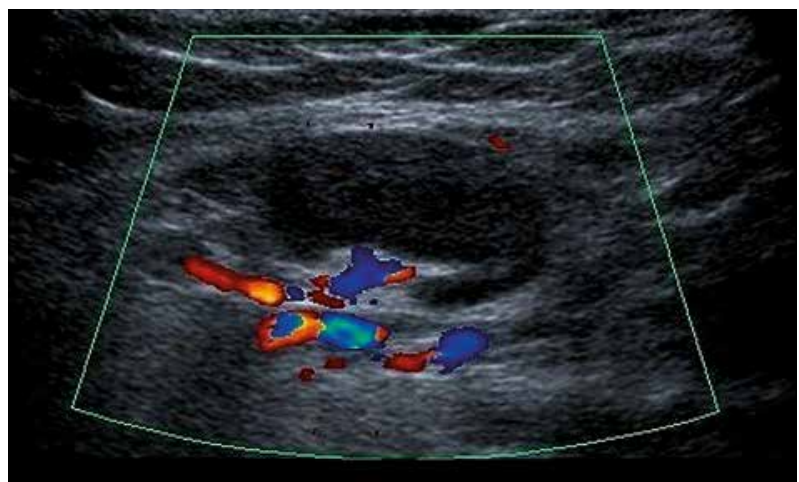

Figure 2. Benign lymph node. A 47-year-old female. Submandibular region. Long axis: $13 \mathrm{~mm}$. Short axis: $5 \mathrm{~mm}$. S/L ratio: 0.4 . Hilar vascularity. Pathology: Reactive hyperplasia 


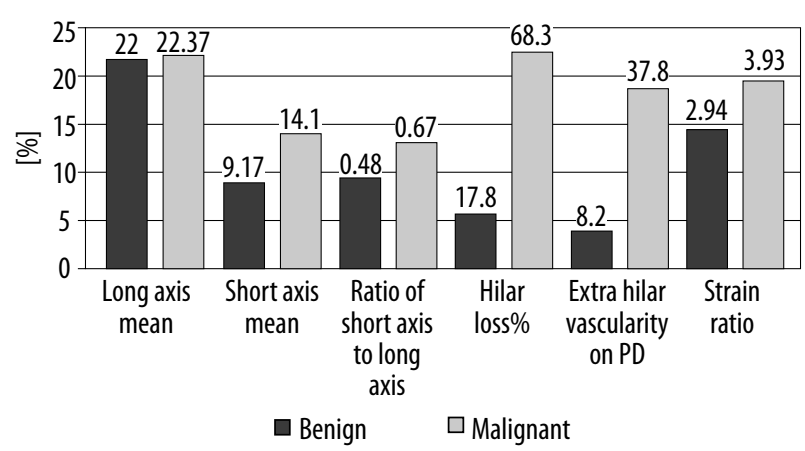

Figure 3. Comparison of conventional ultrasound and strain ratio features between benign and malignant lymph nodes some studies have found them valuable. There is a common presumption that if a lymph node is enlarged it tends to be malignant. On the other hand, our results support articles that state that the short axis, $\mathrm{S} / \mathrm{L}$ ratio, absence of the fatty hilum, and extrahilar vascularity are significant indicators of malignancy. Figures 1 and 2 show malignant and benign lymph node characteristics. Figure 3 summarizes ultrasound and strain-wave elastography findings in benign and malignant lymph nodes.

\section{Conflict of interest}

The authors report no conflict of interest.

\section{References}

1. Ying M, Bhatia KSS, Lee YH, et al. Review of ultrasonography of malignant neck nodes: Greyscale, Doppler, contrast enhancement and elastography. Cancer Imaging 2014; 13: 658-669.

2. Lo WC, Cheng PW, Wang CT, et al. Real-time ultrasound elastography: An assessment of enlarged cervical lymph nodes. Eur Radiol 2013; 23: 2351-2357.

3. Ying L, Hou Y, Zheng HM, et al. Real-time elastography for the differentiation of benign and malignant superficial lymph nodes: A metaanalysis. Eur J Radiol 2012; 81: 2576-2584.

4. Lyshchik A, Higashi T, Asato R, et al. Cervical lymph node metastases: Diagnosis at sonoelastography: Initial experience. Radiology 2007; 243: 258-267.

5. Ishibashi N, Yamagata K, Sasaki H, et al. Real-time tissue elastography for the diagnosis of lymph node metastasis in oral squamous cell carcinoma. Ultrasound Med Biol 2012; 38: 389-395.

6. Alam F, Naito K, Horiguchi J, et al. Accuracy of sonographic elastography in the differential diagnosis of enlarged cervical lymph nodes: Comparison with conventional B-mode sonography. Am J Roentgenol 2008; 191: 604-610.

7. Ying M, Ahuja AT, Evans R, et al. Cervical lymphadenopathy: Sonographic differentiation between tuberculous nodes and nodal metastases from non-head and neck carcinomas. J Clin Ultrasound 1998; 26: 383-389.
8. Ying M, Ahuja A, Brook F, et al. Vascularity and greyscale sonographic features of normal cervical lymph nodes: Variations with nodal size. Clin Radiol 2001; 56: 416-419.

9. Gupta A, Rahman K, Shahid M, et al. Sonographic assessment of cervical lymphadenopathy: Role of high resolution and color doppler imaging. Head Neck 2011; 33: 297-302.

10. Imani Moghaddam M, Davachi B, Mostaan LV, et al. Evaluation of the sonographic features of metastatic cervical lymph nodes in patients with head and neck malignancy. J Craniofac Surg 2011; 22: 2179-2184.

11. Lyshchik A, Higashi T, Asato R, et al. Cervical lymph node metastases: Diagnosis at sonoelastographyinitial experience. Radiology 2007; 243: 258-267.

12. Wu CH, Chang YL, Hsu WC, et al. Usefulness of Doppler spectral analysis and power Doppler sonography in the differentiation of cervical lymphadenopathies. Am J Roentgenol 1998; 171: 503-509.

13. Gronkiewicz J, Vade A. Cervical lymph node fine needle aspiration in patients with no history of malignancy. Ultrasound Q 2013; 29: 323-326.

14. Yeon HY, Kim AJ, Son EJ, et al. Sonographic findings predictive of central lymph node metastasis in patients with papillary thyroid carcinoma. J Ultrasound Med 2013; 32: 2145-2151.

15. Park YM, Fornage BD, Benveniste AP, et al. Strain elastography of abnormal axillary nodes in breast cancer patients does not improve diagnostic accuracy compared with conventional ultrasound alone. Am J Roentgenol 2014; 203: 1371-1378. 